\title{
Checklist of Flowering Plants (Magnoliophyta) of Mount Nglanggeran, Gunungkidul: Confirmation and Update of Flora of Java and APG III
}

\author{
Widodo $^{1}$, Muhammad Jafar Luthfi ${ }^{2}$ \\ ${ }^{1,2}$ Biological Education Department, Faculty of Science and Technology, UIN Sunan Kalijaga \\ J1. Marsda Adisucipto 55281 Yogyakarta, Indonesia \\ Author correspondency: \\ wwidodo594@gmail.com ${ }^{1}$
}

\begin{abstract}
This study aimed to collect data on the species plants in Mount Nglanggeran, to confirm and update the existence of these plants from Flora of Java book by Backer \& Bakhuizen, and to showing the visual data of plant species in Nglanggeran Mountain. This research used survey method through in-situ visit and specimen collection. Monitoring and visits were conducted based on prediction of flowering period and fruit-bearing season. Eighty plant families of flowering plants were found in the mount Nglanggeran. Based on the phylogenetic arrangement of flowering plants it was found that all the main groups (clades) of flowering plants were found at this location.
\end{abstract}

Keywords: checklist; magnoliophyta; update plant list; Mount Nglanggeran; local plant.

\section{INTRODUCTION}

Non-cultivated plants in Java, especially in Yogyakarta tends to be neglected by young generations. The plants left as ornamental plants, wild plant on waste lands or protected places such as in protected forests, cemeteries, etc. More often unknown plant identified only by its scientific name and preserved in herbarium centers in Europe and discussed in old books in the past but the current distribution is almost no longer updated.

Mount Nglanggeran area is now an attractive tourist destination. Mount Nglanggeran is the remnant of ancient volcano with almost parallel magmatic rock area. This area is uninhabited and is a protected area. The status of such area cause the protection of different types of local and wild plants that are no longer in residential areas. In previous research, the author has found plant species that can only be traced from old herbaria and old manuscript sources. Publications and data of such plants are very rare.

Flora of Java (Backer \& Bakhuizen, 1962-70) and Flora of British India (Hooker, 1885) are important manuscripts as guidance for plant identification in Java, Indonesia, and Southeast Asia in general. Not all local plants can be identified and listed in this book, but through comparison based on the description of the family and genus levels can lead identification to other books and herbaria-herbarium that have been published. The greatest disadvantage of these books is that they are filled with verbal descriptions with no illustrations, making it very difficult to identify even though the plant data is relatively complete.
The identification process of plants in mount Nglanggeran is relatively difficult and requires a lot of data. Often the identity of plant species is known for many years after the observation done. Preliminary data from author showed that the mount Nglanggeran plant represents nearly 230 flowering plant families in the Flora of Java (1962-70) from about 560 flowering plant families in the world (Takhtajan, 2008) .

Based on the above description, it is important to document the Nglanggeran plants and systematically arranged the hierarchy of plant families. This systematic visual documentation is useful to help the use of Flora of Java book, to explore and introducing the richness of plants in mount Nglanggeran, and revealing the vegetation data that is no longer recognized by people.

This paper aimed to show the data of families of plant species in mount Nglanggeran, to confirm the existence of the plants in Flora of Java by Backer \& Bakhuizen, and showing visual photograph data of plant species in mount Nglanggeran Mountain.

\section{MATERIALS AND METHODS}

This researchs were field and literature research. Field research was survey method (Singh, 2010. Collection was done by sampling specimen for herbaria and photograping herbarium sampling with attention on the sustainability of plant population. Monitoring and visits are conducted with consideration of the prediction of the period inflorescence and fruit formation. 


\section{Equipments and materials}

Equipments for observation and collection consist of: Sony Nex F3 digital camera, Sony Cyber-Shot DSCW180 digital camera, Canon DSLR digital camera, glass slide, micrometer, slide length, small roll meter, plastic collection, scissors, cutter, GPS (Global Positioning System), dry herbarium collection kit, flakon bottle, Nikon SMZ 1500 stereo microscope equipped with camera, Nikon Eclipse 50 light microscope equipped with Nikon DSF1 camera. Materials for observation and collection consist of: Aquadest, Alcohol 70\%, FAA solution (Formalin Acetic Alchohol).

\section{Work procedure}

The working procedures were as follows: photograping and observing of specimen in situ, herbarium, and flower/fruit. The data were compared to Flora of Java (Backer \& Bakhuizen, 1963-70) and other existing literature, checking and matching with herbarium types and illustrations/drawings in the literature to identify the specimen.

\section{RESULTS AND DISCUSSION}

Flora of Java book by Backer \& Bakhuizen (1963-1968) describes Spermatophyta found in Java. Description is an explanation of important character of plants or groups of plants in the category of family, genus, and species. The pattern of explanation of the characteristics of the plant begins with the description of the characteristics of flowers followed by vegetative characteristics.

Flora of Java book by Backer \& Bakhuizen (19631968) consists of volumes 1, 2, 3. Volumes 1 and 2 contain descriptions of dicotyle plants consisting of 190 families. Volume 3 contains descriptions of monocotyle containing 48 families. So the total family of plants in Flora of Java is 238 families. This book contains 2885 genera on Volume 1, 2199 genera on Volume 2 and 2018 genera on Volume 3, or a total of 7112 genera.

This book is very useful for the identification of plants found in yards, gardens, fields, and forests. The main obstacles to the use and limitations of this book are the absence of visual illustrations and requirement of flower as main character for identification discovery of interest and its characteristics in the identification process. Wild and unknown vegetation in forest areas which is rarely blooming at observation, could be very difficult to identify without the appearance of flowers and fruit.

From this study it is found that the diversity of plant species in the mount Nglanggeran are 80 families of seed plants from 238 families in the book Flora of Java (Backer and Bakhuizen, 1963-1968) or around 33, 19\%. Details of the percentage of family are 32 out of 110 families in the book Volume 1 (29.09\%); 33 of the 80 families in the book Volume 2 (41.25\%); 15 families of 48 families in the book Volume $3(31.25 \%)$.

The existence of flowering plants genera in Mount Nglanggeran are 265 genera of 7112 genera in Flora of Java (Backer and Bakhuizen, 1963-1965) or 3.73\% range. Detail of genera percentage are 108 genera of 2885 genera Volume 1 (3.74\%); 105 of 2199 genera in Volume $2(4,74 \%)$ and 51 of 2018 genera in volume 3 (2.51\%).

Flowering plant is a group of plants that dominate the earth today. Seed plants in old taxonomic terms are manifestations of the Spermatophyta class, consisting of subclass Gymnospermae and Angiospermae (flowering plant). In the book Backer \& Bakhuizen (1963-1968), Gymnospermae consist of 7 families whereas flowering plants consist of 231 families.

Table 1. Percentage of representation of each category of plant classification in mount Nglanggeran.

\begin{tabular}{|c|c|c|c|c|c|}
\hline No. & Clade & $\begin{array}{l}\text { Number of } \\
\text { Orders }\end{array}$ & $\begin{array}{l}\text { Order representation in } \\
\text { mount Nglanggeran }\end{array}$ & Number of family & $\begin{array}{l}\text { Family representation in } \\
\text { Mount Nglanggeran }\end{array}$ \\
\hline 1 & Unrank & 4 & 0 & 7 & 0 \\
\hline 2 & Magnoliids & 4 & 3 or $75 \%$ & 20 & 4 or $20 \%$ \\
\hline 3 & Monocot Commeliniids & 5 & 4 or $80 \%$ & 31 & 7 or $22,58 \%$ \\
\hline 4 & $\begin{array}{l}\text { Monocot Non } \\
\text { Commeliniids }\end{array}$ & 7 & 5 or $71,42 \%$ & 46 & 9 or $19,56 \%$ \\
\hline 5 & Eudicot Unrank & 6 & 1 or $16,67 \%$ & 15 & 2 or $13,33 \%$ \\
\hline 6 & $\begin{array}{l}\text { Core Eudicot Rosids } \\
\text { Fabids }\end{array}$ & 8 & 7 or $87,5 \%$ & 71 & 14 or $19,71 \%$ \\
\hline 7 & $\begin{array}{l}\text { Core Eudicot Rosids } \\
\text { Malvids }\end{array}$ & 6 & 4 or $66,67 \%$ & 59 & 13 or $22,03 \%$ \\
\hline 8 & Core Eudicot Unrank & 7 & 6 or $85,71 \%$ & 87 & 15 or $13,21 \%$ \\
\hline 9 & $\begin{array}{l}\text { Core Eudicot Asterids } \\
\text { Lamids }\end{array}$ & 5 & 4 or $80 \%$ & 40 & 14 or $35 \%$ \\
\hline 10 & $\begin{array}{l}\text { Core Eudicot Asterids } \\
\text { campanulids }\end{array}$ & 7 & 2 or $28,57 \%$ & 27 & 4 or $14,81 \%$ \\
\hline
\end{tabular}


According to Singh (2010), gymnospermae plants consist of 11 families covering 80 genera, whereas flowering plants consist of 485 families covering 13,372 genera including 253,000 species $(10,760$ genera, 196,990 species dikotil, 2,612 genera, 56,310 monocots species).

Currently the reform of the categories of angiospermae plant classification (flowering plants) is carried out by the APG association (Angiospermae Phylogeny Group) (APG III, 2009). Flowering plants comprised about 62 orders that included about 410 families. Table 2 shows the checklist of the existence of plant species in mount Nglanggeran following the family order based on APG III (2009). Table 1 shows the proportions of each category.

From Table 1 it is found that all the major flowering plant clusters are in Nglanggeran Mountain, except for basal groups of Mangnoliids which are present only in certain regions of the world. Based on the percentage of representation of the order, it is found that the plants in Nglanggeran Mountain were mainly clade Core eudicot Rosiid Fabids group followed by Core Eudicot unrank, Core Eudicot Asterids Lamids, Monocot Commeliniids. Based on the percentage of representation of existing families, it is found that the composition of plants in mount Nglanggeran is mainly from Cie Eudicot unrank clade, Core Eudicot Rosiid Fabids, Eudicot Asterids Lamiids, Core Eudicot Rosids Malvids.

The representation of all the major clusters of flowering plants in mount Nglanggeran shows that this location is an important site for continuous researchs. Visual data in the form of specimens, photographs, or living plants in situ can be utilized to introduce more easily to the people about the diversity of flowering plants. Detailed photographs or plant images need to be prepared for this step.

Description of plant character of Flora of Java should be supported by visual data and real specimens in relation to the importance of identifying species for various purposes. The visual data of plants in mount Nglanggeran obtained in this study should be arranged systematically to complement and facilitate the use of the book. Preparation of hierarchy of clade, order, family of flowering plants according to APG III is done to update how to study plant diversity and its identification.

\section{CONCLUSION}

It was found 80 families of flowering plants in the mount Nglanggeran. Based on the phylogenetic arrangement of flowering plants it was found that all the main groups (clades) of flowering plants were found at this location.

\section{ACKNOWLEDGEMENTS}

The authors wish to thank the Society for Research and Community Service UIN Sunan Kalijaga who funded this research, Sugeng Handoko as the manager of ecotourism area Nglanggeran Gunungkidul Mountain Yogyakarta, Bayu Setya Aji, Rendi Yuntara and Didik Zulfahmi Akbar.

\section{REFERENCES}

Backer, C. A. \& Bakhuizen. 1963. Flora of Jawa (Spermatophytes Only). Vol I, II, III. Groningen: N. V. P. Noordhoff.

Birgitta Bremer, Kåre Bremer, Mark W. Chase, Michael F. Fay, James L. Reveal, Douglas E. Soltis, Pamela S. Soltis and Peter F. Stevens. 2009. An update of the Angiosperm Phylogeny Group classification for the orders and families of flowering plants: APG III. Botanical Journal of the Linnean Society, 2009, 161, 105-121.

CBD (Convention on Biological Diversity). 2002. Strategi Global Konservasi Tumbuhan (Translated). Bogor: Botanic Garden Conservation International.

Heim E. 2015. Flora and Vegetation of Bali Indonesia. Herstellung und Verlag, Norderstedt.

Hooker, J. D. 1885. Flora of British India (Vol. I, II, III, IV). London: Reeve and Co.

Keng H. 1990. The Consist Flora of Singapore. Singapore University Press, Singapore.

Knowles, B. 2008. Systematics and Taxonomy Response to the House of Lord Science and Technology Committee. United Kingdom: Bioscience Federation.

Ng, F. S. P. 1972-73. Tree Flora of Malaya Vol. 3. Syarikat Seng Teik, Selangor.

Quattrocchi, U. 2012. CRC Word Dictionary of Medicinal and Poisonous Plants. Comon Names, Scientific Names, Eponyms, Synonims and Etymology. CRC Press, London

Randler, C. 2008. Teaching Species Identification, A Prerequisite for Learning Biodiversity and Understanding Ecology. Eurasia Journal of Mathematics, Science \& Technology Education, 4 (3):223-231.

Randler, C., and Bogner, F. X. 2006. Cognitive Achievement in Identification Skills. Journal of Biology Education, 40 (4): 161-165.

Rustaman, N. Y. 2006. Arah Pembelajaran Taksonomi Tumbuhan dan Assesmennya di LPTK dan Sekolah (Online), http:/www.file.upi.edu), Retrieved at 23 June 2012.

Simpson, M.G. 2006. Plant Systematics. Amsterdam: Elsevier Academic Press.

Singh, G. 2010. Plant Systematics. New Hampshire: Science Publisher.

Takhtajan, A. 2009. Flowering Plant. St Petersburg: Springer. 
Table Family plant list (Backer \& Bakhuizen, 1962) and list of seed plants in mount Nglanggeran.

\begin{tabular}{|c|c|c|c|c|c|c|c|}
\hline No. & Family & $\begin{array}{l}\text { Availability } \\
\text { in location }\end{array}$ & $\begin{array}{l}\text { Estimation } \\
\text { of number }\end{array}$ & Species Name & $\begin{array}{l}\text { Availability } \\
\text { in location }\end{array}$ & $\begin{array}{l}\text { Estimation } \\
\text { of number }\end{array}$ & Species Name \\
\hline 1 & Magnoliaceae & & & & Available & 1 & Magnolia alba \\
\hline 2 & Schisandraceae & & & & & & \\
\hline 3 & Annonaceae & Available & 6 & $\begin{array}{l}\text { Uvaria rufa } \\
\text { Anomianthus dulcis } \\
\text { Meiogyne sp } \\
\text { Polyaulax sp } \\
\text { Annona muricata } \\
\text { Annona squamosa }\end{array}$ & Available & 2 & $\begin{array}{l}\text { Stelechocarpus burahol } \\
\text { Polyaltia sp }\end{array}$ \\
\hline 4 & Lauraceae & Available & 2 & Litsea chinensis & Available & 1 & Persea americana \\
\hline 5 & Hernandiaceae & & & & & & \\
\hline 6 & Myristicaceae & & & & Available & 1 & Myristica sp \\
\hline 7 & Ranunculaceae & Available & 1 & Clematis javana & & & \\
\hline 8 & Ceratophyllaceae & & & & & & \\
\hline 9 & Nymphaeaceae & & & & & & \\
\hline 10 & Berberidacaceae & & & & & & \\
\hline 11 & Menispermaceae & Available & 6 & $\begin{array}{l}\text { Pycnarhena montana } \\
\text { Arcangelisia sp } \\
\text { Tinospora coriacea } \\
\text { Stephania hernandifolia } \\
\text { Cissampelos sp } \\
\text { Pericampylus glaucus } \\
\text { Cyclea barbata }\end{array}$ & Available & 1 & Tinospora crispa \\
\hline 12 & Aristolochiaceae & Available & 1 & Aristolochia indica & & & \\
\hline 13 & Raflesiaceae & & & & & & \\
\hline 14 & Nepenthaceae & & & & & & \\
\hline 15 & Piperaceae & Available & 7 & $\begin{array}{l}\text { Piper bettle } \\
\text { Piper retrofractum } \\
\text { Piper nigrum } \\
\text { Piper aduncum } \\
\text { Peperomia pelucida } \\
\text { Peperomia sp } \\
\text { Piper sp }\end{array}$ & & & \\
\hline 24 & Saururaceae & & & & & & \\
\hline 25 & Chloranthaceae & & & & & & \\
\hline 26 & Papaveraceae & & & & & & \\
\hline 27 & Fumariaceae & & & & & & \\
\hline 28 & Turneraceae & & & & & & $\begin{array}{l}\text { Piriqueta racemosa } \\
\text { Turnera ulmifolia }\end{array}$ \\
\hline 29 & Loasaceae & & & & & & \\
\hline 30 & Capparaceae & Available & 5 & $\begin{array}{l}\text { Capparis micracantha } \\
\text { Capparis pyrifolia } \\
\text { Capparis sepiaria } \\
\text { Cleome rutidosperma } \\
\text { Gynandropsis gynandara }\end{array}$ & & & $\begin{array}{l}\text { Cleome sp } \\
\text { Gynandropsis sp }\end{array}$ \\
\hline 31 & Moringaceae & & & & & & Moringa oleifera \\
\hline 32 & Brassicaceae & & & & & & \\
\hline 33 & Violaceae & & & & & & \\
\hline 34 & Resedaceae & & & & & & \\
\hline 35 & Polygalaceae & & 2 & $\begin{array}{l}\text { Polygala paniculata } \\
\text { Polygala glomerata }\end{array}$ & & & \\
\hline 36 & Crassulaceae & & & & & & Kalanchoe pinnata \\
\hline 37 & Saxifragaceae & & & & & & \\
\hline 38 & Droseraceae & & & & & & \\
\hline 39 & Podostemataceae & & & & & & \\
\hline 40 & Elatinaceae & & & & & & \\
\hline 41 & Caryophyllaceae & & & & & & \\
\hline 42 & Molluginaceae & & 1 & & & & $\begin{array}{l}\text { Mollugo pentaphylla } \\
\text { Glinus lotoides (bawukan } \\
\text { berbulu) } \\
\text { Glinus oppositifolius } \\
\text { (bawukan licin) }\end{array}$ \\
\hline 43 & Ficoidaceae & & & & & & Trianthema portulacastrum \\
\hline 44 & Portulacaceae & & 1 & & & & Portulaca oleracea \\
\hline
\end{tabular}


Talinum paniculatum

Talinum fruticosum

Famili baru: Talinaceae

45 Polygonaceae

Polygonum orientale

Polygonum chinense

Polygonum barbatum

Antigonon leptopus

Muehlenbeckia platyclada

\begin{tabular}{cl}
\hline 46 & Phytolaccaceae \\
\hline 47 & Chenopodiaceae \\
\hline 48 & Amaranthaceae
\end{tabular}

Rivinia humilis

Celosia argentea

Amaranthus hybridus

Amaranthus gracilis

Amaranthus spinosus

Cyathula prostrata

Aerva sanguinolenta

Achyranthea aspera

Alternanthera sessilis

Alternanthera

phyloxeroides

Gomphrena celosioides

\begin{tabular}{cl}
\hline 49 & Basellaceae \\
\hline 50 & Linaceae \\
\hline 51 & Zygophyllacea \\
\hline 52 & Geraniaceae \\
\hline 53 & Oxalidaceae
\end{tabular}

Basella rubra

Anredera cordifolia

\begin{tabular}{|c|c|c|c|c|}
\hline 53 & Oxalidaceae & 3 & $\begin{array}{l}\text { Oxalis barrelieri } \\
\text { Oxalis corniculata } \\
\text { Biophytum reinwardtii }\end{array}$ & $\begin{array}{l}\text { Averrhoa carambola } \\
\text { Averrhoa bilimbi }\end{array}$ \\
\hline 54 & Tropaeolaceae & & & \\
\hline 55 & Balsaminaceae & 1 & Impatien platypetala & \\
\hline 56 & Lythraceae & 1 & Lawsonia inermis & $\begin{array}{l}\text { Lagerstroemia indica } \\
\text { Lagerstroemia speciosa } \\
\text { Cuphea hyssopifolia }\end{array}$ \\
\hline 57 & Crypteroniaceae & & & \\
\hline 58 & Sonneratiaceae & & & \\
\hline 59 & Punicaceae & & & $\begin{array}{l}\text { Punica granatum } \\
\text { (Famili Lythraceae) }\end{array}$ \\
\hline 60 & Onagraceae & & & \\
\hline 61 & Trapaceae & & & \\
\hline 62 & Haloragaceae & & & \\
\hline 63 & Callitrichaceae & & & \\
\hline 64 & Thymelaeaceae & 1 & Phaleria octandra & Phaleria macrocarpa \\
\hline 65 & Nyctaginaceae & 3 & $\begin{array}{l}\text { Mirabilis jalapa } \\
\text { Boerhavia erecta } \\
\text { Bougainvillea spectabili }\end{array}$ & \\
\hline
\end{tabular}

\begin{tabular}{|c|c|c|c|c|}
\hline 66 & Proteaceae & & & \\
\hline 67 & Dilleniaceae & 1 & Tetracera scandens & \\
\hline 68 & Pittosporaceae & & & \\
\hline 69 & Bixaceae & & & Bixa orellana \\
\hline 70 & Cochlospermaceae & & & \\
\hline 71 & $\begin{array}{l}\text { Flacourtiaceae } \\
\text { (Salicaceae) }\end{array}$ & 1 & Flacourtia indica & \\
\hline 72 & Tamaricaceae & & & \\
\hline 73 & Passifloraceae & 2 & $\begin{array}{l}\text { Passiflora foetida } \\
\text { Passiflora edulis }\end{array}$ & $\begin{array}{l}\text { Passiflora suberosa } \\
\text { Passiflora vitifolia } \\
\text { Passiflora quadrangularis }\end{array}$ \\
\hline 74 & Cucurbitaceae & 3 & $\begin{array}{l}\text { Momordica charantia } \\
\text { Trichosanthes villosa } \\
\text { Trichosanthes tricuspidata }\end{array}$ & $\begin{array}{l}\text { Citrulus vulgaris } \\
\text { Cucumis sativus } \\
\text { Luffa acutangula } \\
\text { Cucurbita moschata } \\
\text { Coccinia grandis } \\
\text { Sechium edule } \\
\text { Benincasa hispida } \\
\end{array}$ \\
\hline 75 & Begoniaceae & & & \\
\hline 76 & Datiscaceae & & & \\
\hline 77 & Caricaceae & & & Carica papaya \\
\hline 78 & Cactaceae & & & Pereskia sp \\
\hline
\end{tabular}

Nopalea sp 


\begin{tabular}{|c|c|c|c|c|}
\hline & & & & $\begin{array}{l}\text { Opuntia sp } \\
\text { Cereus sp } \\
\text { Hylocereus sp (Buah } \\
\text { Naga) } \\
\text { Epyphyllum sp } \\
\text { (Wijayakusuma) }\end{array}$ \\
\hline 79 & Theaceae & & & \\
\hline 80 & Actinidaceaea & & & \\
\hline 81 & Saurauiaceae & & & \\
\hline 82 & Ocnaceae & & & \\
\hline 83 & Dipterocarpaceae & & & \\
\hline 84 & Myrtaceae & 4 & $\begin{array}{l}\text { Psidium guajava } \\
\text { Zyzygium cumini } \\
\text { Zyzigium polyanthum } \\
\text { Zyzigium javanicum }\end{array}$ & $\begin{array}{l}\text { Zyzygium aromaticum } \\
\text { Zyzigium malaccensis } \\
\text { Melaleuca leucadendron } \\
\text { Callistemon sp }\end{array}$ \\
\hline 85 & Lecythidaceae & 1 & Barringtonia asiatica & $\begin{array}{l}\text { Barringtonia racemosa } \\
\text { Barringtonia asiatica }\end{array}$ \\
\hline 86 & Melastomataceae & 3 & $\begin{array}{l}\text { Melastoma malabathricum } \\
\text { Osbeckia chinensis } \\
\text { Memecylon caeruleum }\end{array}$ & \\
\hline 87 & Combretaceae & 1 & Terminalia catappa & $\begin{array}{l}\text { Quisqualis indikca } \\
\text { (Srigading) }\end{array}$ \\
\hline 88 & Rhizophoraceae & & & \\
\hline 89 & Hyericaceae & & & \\
\hline 90 & Clusiaceae & 2 & $\begin{array}{l}\text { Calophyllum inophyllum } \\
\text { Garcinia mangostana }\end{array}$ & \\
\hline 91 & Tiliaceae & & $\begin{array}{l}\text { Triumfetta indica } \\
\text { Schoutenia ovata } \\
\text { Mutingia calabura }\end{array}$ & Corchorus acutangulus \\
\hline 92 & Elaeocarpaceae & 1 & Mutingia calabura & \\
\hline 93 & Gonystylaceae & & & \\
\hline 94 & Sterculiaceae & 1 & Helicteres hirsuta & Helicteres isora \\
\hline 95 & Bombacaceae & & Ceiba petandra & $\begin{array}{l}\text { Durio zibethinus } \\
\text { Bombax ceiba }\end{array}$ \\
\hline 96 & Malvaceae & 8 & $\begin{array}{l}\text { Sida cordata } \\
\text { Triumfetta indica } \\
\text { Abutilon crispum } \\
\text { Abutilon hirtum } \\
\text { Wissadula periplocifolia } \\
\text { Sida rhombifolia } \\
\text { Sida acuta } \\
\text { Hisbiscus surattensis } \\
\text { Abelmoschus moschatus }\end{array}$ & $\begin{array}{l}\text { Corchorus acutangulus } \\
\text { Melochia corchorifolia } \\
\text { Waltheria americana } \\
\text { Hisbiscus rosa-sinensia } \\
\text { Hisbiscus tiliaceus } \\
\text { Malvaviscus arboreus }\end{array}$ \\
\hline 97 & Malpighiaceae & & & \\
\hline 98 & Erythroxylaceae & & & \\
\hline 99 & Euphorbiaceae & 20 & $\begin{array}{l}\text { Glochidion eriocarpum } \\
\text { Glochidion puberum } \\
\text { Glochidion rubrum } \\
\text { Breynia oblongifolia } \\
\text { Phyllanthus muriculatus } \\
\text { Phyllanthus reticulatus } \\
\text { Phyllanthus emblica } \\
\text { Phyllanthus niruri } \\
\text { Sauropus androgynus } \\
\text { Bridelia micrantha } \\
\text { Bridelia stipularis } \\
\text { Croton hyrtus } \\
\text { Acalypha indica } \\
\text { Acalypha boehmerioides } \\
\text { Jatropha gossypifolia } \\
\text { Jatropha multifida } \\
\text { Euphorbia hirta } \\
\text { Euphorbia prostrata } \\
\text { Euphorbia heterophylla } \\
\text { Manihot esculenta } \\
\text { Manihot glaziovii } \\
\text { Hevea brasiliensis }\end{array}$ & $\begin{array}{l}\text { Croton variegatus } \\
\text { Acalypha wilkesiana } \\
\text { Ricinus communis } \\
\text { Jatropha curcas } \\
\text { Codiaeum variegatum } \\
\text { Pedilanthus variegatus }\end{array}$ \\
\hline 100 & Daphniphyllaceae & & & \\
\hline 101 & Cunoniaceae & & & \\
\hline 102 & Escalloniaceae & & & \\
\hline
\end{tabular}




\begin{tabular}{|c|c|c|c|c|c|}
\hline 104 & Rosaceae & Available & 1 & Rubus moluccanus & \\
\hline 105 & Dichapetalaceae & & & & \\
\hline 106 & Caesalpiniaceae & Available & 3 & $\begin{array}{l}\text { Cassia siamea } \\
\text { Cassia occidentalis } \\
\text { Cassia obtusifolia }\end{array}$ & \\
\hline 107 & Mimosaceae & Available & 8 & $\begin{array}{l}\text { Albizia montana } \\
\text { Albizia lebbeck } \\
\text { Albizia procera } \\
\text { Leucaena glauca } \\
\text { Mimosa pdica } \\
\text { Mimosa invisa } \\
\text { Acacia auriculiformis } \\
\text { Parkia speciosa }\end{array}$ & \\
\hline 108 & Papilionaceae & Available & 15 & $\begin{array}{l}\text { Crotalaria usaramoensis } \\
\text { Crotalaria striata } \\
\text { Indiofera sumatrana } \\
\text { Desmodium pulchellum } \\
\text { Desmodium gangeticum } \\
\text { Desmodium triflorum } \\
\text { Alysicarpus } \\
\text { nummularifolius } \\
\text { Uraria crinita } \\
\text { Uraria logopoides } \\
\text { Abrus precatorius } \\
\text { Centrosema pubescens } \\
\text { Mucuna pruriens } \\
\text { Flemingia strobilifera } \\
\text { Alysicarpus sp } \\
\text { Gliricidia sepium }\end{array}$ & \\
\hline 109 & Hamamelidaceae & & & & \\
\hline 110 & Buxaceae & & & & \\
\hline 111 & Salicaceae & & & Flacuortia indica & \\
\hline 112 & Myricaceae & & & & \\
\hline 113 & Betulaceae & & & & \\
\hline 114 & Fagaceae & & & & \\
\hline 115 & Casuarinaceae & & & & $\begin{array}{l}\text { Casuarina junghuhnia } \\
\text { Casuarina equisetifolia }\end{array}$ \\
\hline 116 & Ulmaceae & & & & \\
\hline 117 & Moraceae & Available & 16 & $\begin{array}{l}\text { Fatoua sp } \\
\text { Morus sp } \\
\text { Malaisa scandens } \\
\text { Streblus asper } \\
\text { Streblus taxoides } \\
\text { Maclura cochinchinensis } \\
\text { Ficus benyamina } \\
\text { Ficus septica } \\
\text { Ficus montana } \\
\text { Artocarpus integra } \\
\text { Poikilospermum } \\
\text { suaveolens }\end{array}$ & \\
\hline 118 & Urticaceaea & Available & & $\begin{array}{l}\text { Laportea sp } \\
\text { Fleurya sp } \\
\text { Pilea microphyla } \\
\text { Pouzolzia zeylanica } \\
\text { Boehmeria sp } \\
\end{array}$ & \\
\hline 119 & Cannabaceae & & & & \\
\hline 120 & Aquifoliaceae & & & & \\
\hline 121 & Celastraceae & Available & 1 & Celastrus scandens & \\
\hline 122 & Hippocrateaceae & & & & \\
\hline 123 & Icacinaceae & & & & \\
\hline 124 & Salvadoraceae & & & & \\
\hline 125 & Olacaceae & Available & 1 & Olax scandes & \\
\hline 126 & Opiliaceae & & & & \\
\hline 127 & Loranthaceae & Available & 1 & Elythranthe sp & \\
\hline 128 & Santalaceae & Available & 1 & Santalum album & \\
\hline 129 & Balanophoraceae & & & & \\
\hline 130 & Rhamnaceaea & Available & 2 & Zizyphus oenoplia & \\
\hline 131 & Elaeagnaceae & & & & \\
\hline 132 & Vitaceae & Available & 7 & $\begin{array}{l}\text { Vitis discolor } \\
\text { Tetrastigma }\end{array}$ & \\
\hline
\end{tabular}




\begin{tabular}{|c|c|c|c|c|c|}
\hline & & & & $\begin{array}{l}\text { leucostaphylum } \\
\text { Cissus repens } \\
\text { Cayratia trifolia } \\
\text { Leea aequata } \\
\text { Leea rubra }\end{array}$ & \\
\hline 133 & Rutaceae & & 5 & $\begin{array}{l}\text { Glycosmis petaphylla } \\
\text { Murraya paniculata } \\
\text { Clausena excavata } \\
\text { Aegle marmelos } \\
\text { Zanthoxylum sp } \\
\end{array}$ & \\
\hline 134 & Simarubaceae & Available & 1 & Brucea javanica & \\
\hline 135 & Burseraceae & & & & \\
\hline 136 & Meliaceae & Available & 2 & $\begin{array}{l}\text { Swietenia mahagoni } \\
\text { Chisocheton sp }\end{array}$ & $\begin{array}{l}\text { Lansium domesticum } \\
\text { Melia azedarach } \\
\text { Dysoxylum sp. }\end{array}$ \\
\hline 137 & Sapindaceae & Available & 3 & $\begin{array}{l}\text { Cardiospermum } \\
\text { halicacabum } \\
\text { Allophylus cobbe } \\
\text { Erioglossum rubiginosum }\end{array}$ & $\begin{array}{l}\text { Shcleicera oleosa } \\
\text { Euphoria longana } \\
\text { Nephelium lappaceum } \\
\text { Pometia pinnata } \\
\text { Filicium decipiens }\end{array}$ \\
\hline 138 & Aceraceae & & & & \\
\hline 139 & Sabiaceae & & & & \\
\hline 140 & Staphyleaceaea & & & & \\
\hline 141 & Anacardiaceae & Available & 4 & $\begin{array}{l}\text { Anacardium occidentale } \\
\text { Mangifera indica } \\
\text { Mangifera odorata } \\
\text { Gluta renghas }\end{array}$ & $\begin{array}{l}\text { Spondias dulcis } \\
\text { Lannea coromandeca }\end{array}$ \\
\hline 142 & Connaraceae & & & & \\
\hline 143 & Juglandaceae & & & & \\
\hline 144 & Cornaceae & & & & \\
\hline 145 & Alangiaceae & & & & \\
\hline 146 & Nyssaceae & & & & \\
\hline 147 & Araliaceae & Available & 1 & Schefflera sp & $\begin{array}{l}\text { Nothopanax scutellarium } \\
\text { Polyscias sp } \\
\text { Arthrophyllum sp }\end{array}$ \\
\hline 148 & Apiaceae & Available & 2 & $\begin{array}{l}\text { Centela asiatica } \\
\text { Eringium foetidum }\end{array}$ & Hydrocotyle sp \\
\hline 149 & Clethraceae & & & & \\
\hline 150 & Ericaceaea & & & & \\
\hline 151 & Vacciniaceae & & & & \\
\hline 152 & Epacridaceae & & & & \\
\hline 153 & Ebenaceae & Available & 1 & Diospyros truncata & \\
\hline 154 & Sapotaceae & & & & $\begin{array}{l}\text { Crysophyllum cainito } \\
\text { Mimusops elingi } \\
\text { Manilkara kauki } \\
\text { Manilkara achras }\end{array}$ \\
\hline 155 & Myrisinaceae & Available & 2 & $\begin{array}{l}\text { Ardisia humilis } \\
\text { Ardisia crenata }\end{array}$ & \\
\hline 156 & Styracaceae & & & & \\
\hline 157 & Symplocaceae & & & & \\
\hline 158 & Loganiaceae & Available & 2 & $\begin{array}{l}\text { Spigelia althemia } \\
\text { Fagraea ceilanica }\end{array}$ & \\
\hline 159 & Oleaceae & Available & 1 & Jasminum pubescens & \\
\hline 160 & Apocynaceae & Available & 8 & $\begin{array}{l}\text { Alstonia sholaris } \\
\text { Alstonia angustiloba } \\
\text { Rauvolfia verticilata } \\
\text { Anodendron paniculatum } \\
\text { Chonemorpha fragran } \\
\text { Ichnocarpus frutescens } \\
\text { Tabernaemontana } \\
\text { macrocarpa } \\
\text { Wrightia pubescens }\end{array}$ & \\
\hline 161 & Asclepiadaceae & Available & 6 & $\begin{array}{l}\text { Cryptolepis sinensis } \\
\text { Calotropis gigantea } \\
\text { Hoya sp } \\
\text { Marsdenia brunoniana } \\
\text { Telosma puberula } \\
\text { Cosmostigma racemosum }\end{array}$ & $\begin{array}{l}\text { Cynanchum sp } \\
\text { Marsdenia tenacissima } \\
\text { Gymnema sylvestris } \\
\text { Asterostemma repandum }\end{array}$ \\
\hline
\end{tabular}




\begin{tabular}{|c|c|c|c|c|}
\hline & & & & $\begin{array}{l}\text { Ophiorrhiza mungos } \\
\text { Nauclea orientalis } \\
\text { Musaenda frondosa } \\
\text { Pavetta indica } \\
\text { Psychotria sp } \\
\text { Paederia scandens } \\
\text { Vangueria spinosa-Meyna } \\
\text { grisea }\end{array}$ \\
\hline 163 & Capriofoliaceae & & & \\
\hline 164 & Valerianaceae & & & \\
\hline 165 & Dipsaceae & & & \\
\hline 166 & Asteraceae & Available & 14 & $\begin{array}{l}\text { Vernonia cinerea } \\
\text { Elephantopus scaber } \\
\text { Pseudoelephantopus } \\
\text { spicatus } \\
\text { Ageratum conyzoides } \\
\text { Euphatorium inulifolium } \\
\text { Erigeron sumatrensis } \\
\text { Eclipta prostrata } \\
\text { Wedelia montana } \\
\text { Wedelia biflora } \\
\text { Synedrella nodiflora } \\
\text { Bidens biternata } \\
\text { Tridax procumbens } \\
\text { Emilia sonchifolia } \\
\end{array}$ \\
\hline 167 & Gentianaceae & Available & 1 & Isotoma longiflora \\
\hline 168 & Primulaceae & & & \\
\hline 169 & Plumbaginaceae & Available & 1 & Plumbago zeylanica \\
\hline 170 & Plantaginaceae & & & \\
\hline 171 & Campanulaceae & & & \\
\hline 172 & Sphenocleaceae & & & \\
\hline 173 & Lobeliaceae & & & \\
\hline 174 & Goodeniaceae & & & \\
\hline 175 & Stylidiaceae & & & \\
\hline 176 & Polemoniaceae & & & \\
\hline 177 & Hydrophyllaceae & & & \\
\hline 178 & Boraginaceae & Available & 2 & $\begin{array}{l}\text { Ehretia microphylla } \\
\text { Heliotropium indicum }\end{array}$ \\
\hline 179 & Solanaceae & & 4 & $\begin{array}{l}\text { Physalis minima } \\
\text { Solanum torvum } \\
\text { Solanum comitis } \\
\text { Solanum nigrum }\end{array}$ \\
\hline 180 & Convolvulaceae & Available & 5 & $\begin{array}{l}\text { Merremia hastata } \\
\text { Argyreia mollis }\end{array}$ \\
\hline 181 & Scrophulariaceae & Available & 3 & $\begin{array}{l}\text { Lingdernia crustacea } \\
\text { Scoparia dulcis }\end{array}$ \\
\hline 182 & Orobanchaceae & & & \\
\hline 183 & Lentibulariaceaea & & & \\
\hline 184 & Gesneriaceae & Available & 1 & Epithema horsfieldii \\
\hline 185 & Bignoniaceae & Available & 2 & $\begin{array}{l}\text { Oroxylum indicum } \\
\text { Crescentia cujete }\end{array}$ \\
\hline 186 & Pedaliaceae & & & \\
\hline 187 & Acanthaceae & Available & 6 & $\begin{array}{l}\text { Thunbergia fragrans } \\
\text { Andrographis paniculata } \\
\text { Ruelia napifera } \\
\text { Strobilanthes crispus } \\
\text { Asystasia gangetica }\end{array}$ \\
\hline 188 & Myoporaceae & & & \\
\hline 189 & Verbenaceae & Available & 9 & $\begin{array}{l}\text { Tectona grandis } \\
\text { Lantana camara } \\
\text { Stachytarpeta jamaicensis } \\
\text { Vitex sp } \\
\text { Cleroderdrum serratum } \\
\text { Clerodendrum inerme } \\
\text { Vitex sp } \\
\text { Duranta erecta } \\
\text { Premna odorata }\end{array}$ \\
\hline 190 & Lamiaceae & Available & 4 & $\begin{array}{l}\text { Leucas lavandulifolia } \\
\text { Salvia riparia }\end{array}$ \\
\hline
\end{tabular}




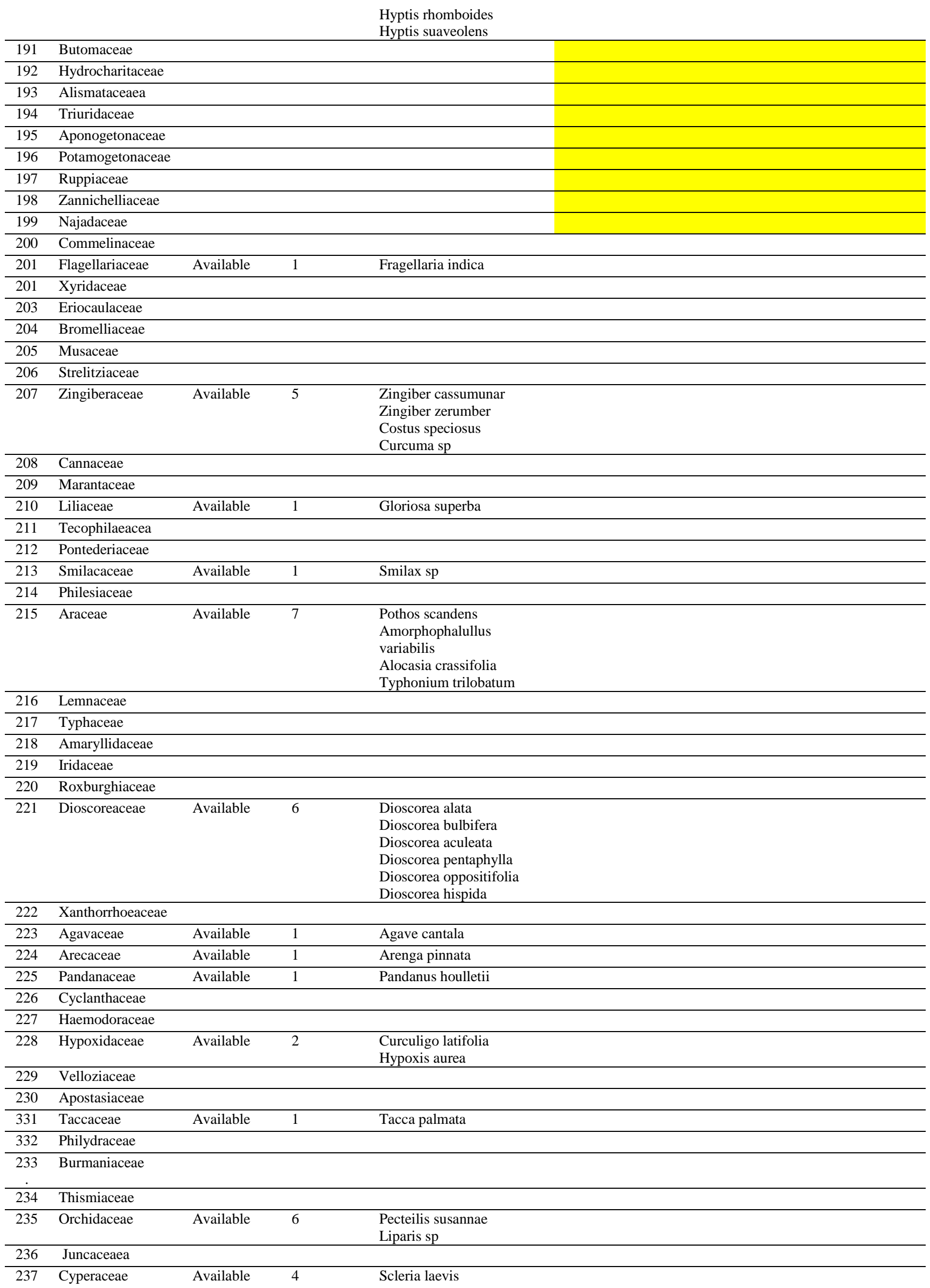




238 Poaceae $\quad 14 \quad \begin{aligned} & \text { Imperata cylindrica } \\ & \text { (Alang-alang) } \\ & \text { Pollinia ciliata } \\ & \text { Polytrias amaura (Rumput } \\ & \text { Lamuran) } \\ & \text { Pogonatherum paniceum } \\ & \text { (Rumput Wesen) } \\ & \text { Andropogon aciculatus } \\ & \text { (Rumput Jarum) } \\ & \text { Themeda arguens (Rumput } \\ & \text { Merak) } \\ & \text { Oplesmenus compositus } \\ & \text { Setaria sp } \\ & \text { Axonopphus compressus } \\ & \text { Anastrophus compressus }\end{aligned}$

Table Checklist of exist plant species in mount Nglanggeran by Clade, Order, Family as suggested by APG III (2009).

\begin{tabular}{|c|c|c|c|c|}
\hline \multicolumn{2}{|l|}{ CLADE } & ORDER & FAMILY & $\begin{array}{l}\text { EXSISTENCE IN } \\
\text { MOUNT } \\
\text { NGLANGGERAN }\end{array}$ \\
\hline \multicolumn{5}{|c|}{ Amborellaceae } \\
\hline & & \multirow[t]{3}{*}{ Nymphaeales } & \multirow{3}{*}{\multicolumn{2}{|c|}{$\begin{array}{l}\text { Cabombaceae } \\
\text { Hydatellaceae } \\
\text { Nymphaeaceae } \\
\end{array}$}} \\
\hline & & & & \\
\hline & & & & \\
\hline & & \multirow[t]{3}{*}{ Austrobaileyales } & \multirow{3}{*}{\multicolumn{2}{|c|}{$\begin{array}{l}\text { Austrobaileyaceae } \\
\text { Schisandraceae+Illiciaceae } \\
\text { Trimeniaceae }\end{array}$}} \\
\hline & & & & \\
\hline & & & & \\
\hline & & Cloranthales & Chloranthaceae & \\
\hline \multirow{20}{*}{\multicolumn{2}{|c|}{ MAGNOLIIDS }} & \multirow[t]{5}{*}{ Piperales } & Aristolochiaceae & $\mathrm{v}$ \\
\hline & & & Hydnoraceae & \\
\hline & & & Lactoridaceae & \\
\hline & & & Piperaceae & $\mathrm{v}$ \\
\hline & & & Saururaceae & \\
\hline & & \multirow[t]{2}{*}{ Canellales } & Canellaceae & \\
\hline & & & Winteraceae & \\
\hline & & \multirow[t]{6}{*}{ Magnoliales } & Annonaceae & $\mathrm{v}$ \\
\hline & & & Deneriaceae & \\
\hline & & & Eupomatiaceae & \\
\hline & & & Himantandraceae & \\
\hline & & & Magnoliaceae & \\
\hline & & & Mytisticaceae & \\
\hline & & \multirow[t]{7}{*}{ Laurales } & Atherospermataceae & \\
\hline & & & Calycanthaceae & \\
\hline & & & Gomortegaceae & \\
\hline & & & Hernandiaceae & \\
\hline & & & Lauraceae & $\mathrm{v}$ \\
\hline & & & Monimiaceae & \\
\hline & & & Siparunaceae & \\
\hline \multirow[t]{11}{*}{ MONOCOT } & \multirow[t]{11}{*}{ Commelinids } & \multirow[t]{5}{*}{ Commelinales } & Commelinaceae & $\mathrm{v}$ \\
\hline & & & Haemodoraceae & \\
\hline & & & Hanguanaceae & \\
\hline & & & Philydraceae & \\
\hline & & & Pontederiaceae & \\
\hline & & \multirow[t]{6}{*}{ Zingiberales } & Cannaceae & \\
\hline & & & Costaceae & $\mathrm{v}$ \\
\hline & & & Heliconiaceae & \\
\hline & & & Lowiaceae & \\
\hline & & & Marantaceae & \\
\hline & & & Musaceae & \\
\hline
\end{tabular}




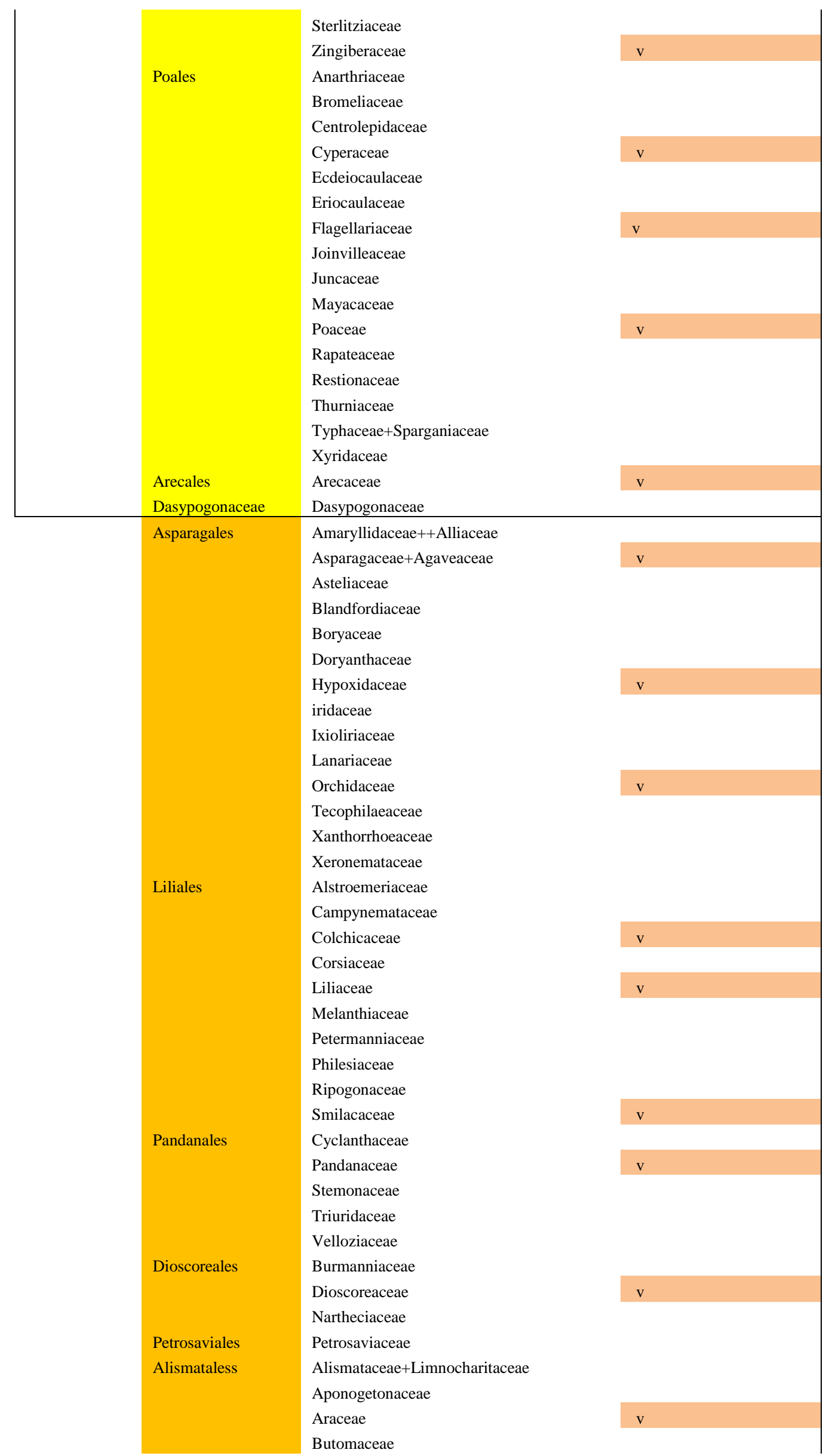




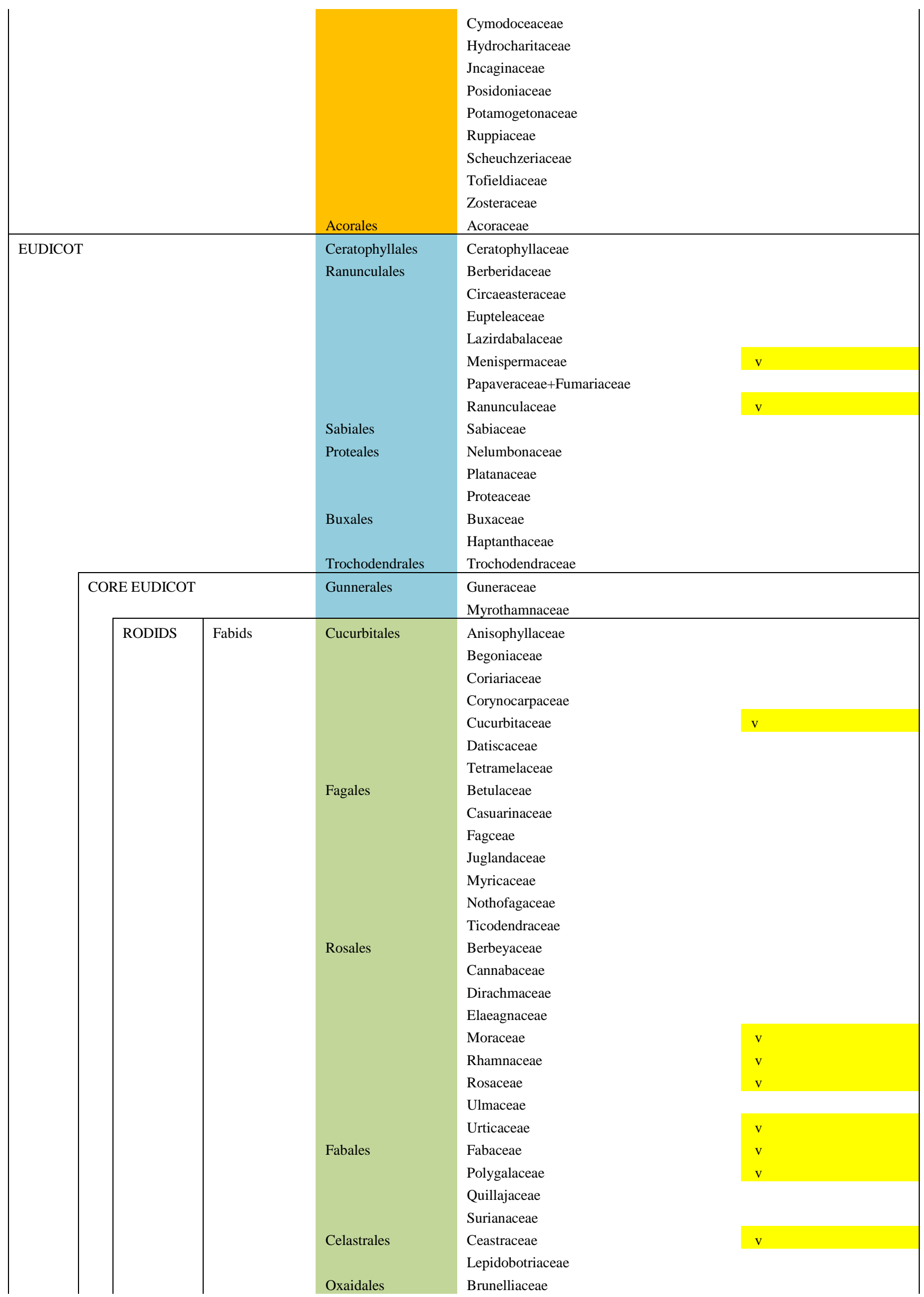




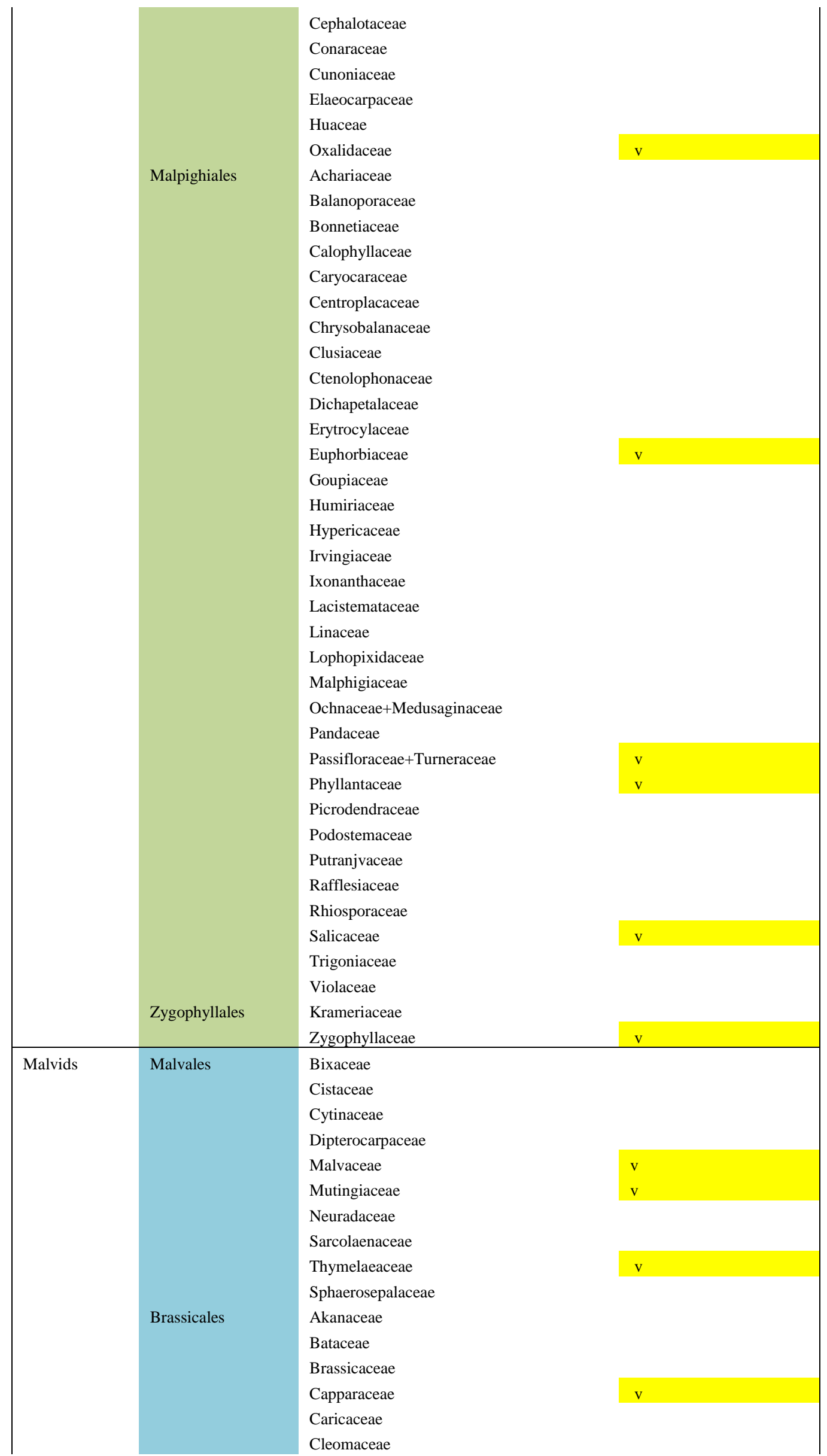




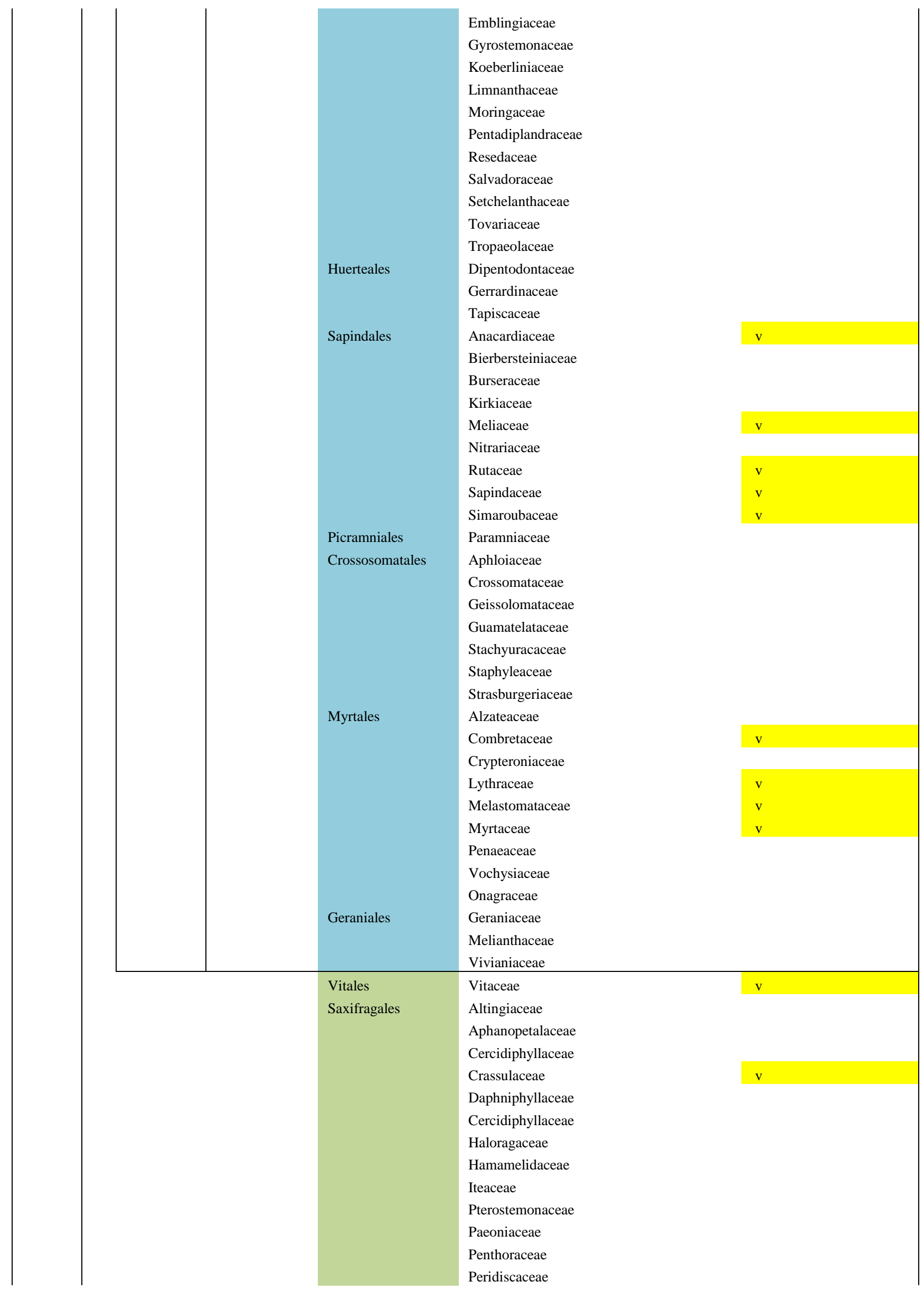



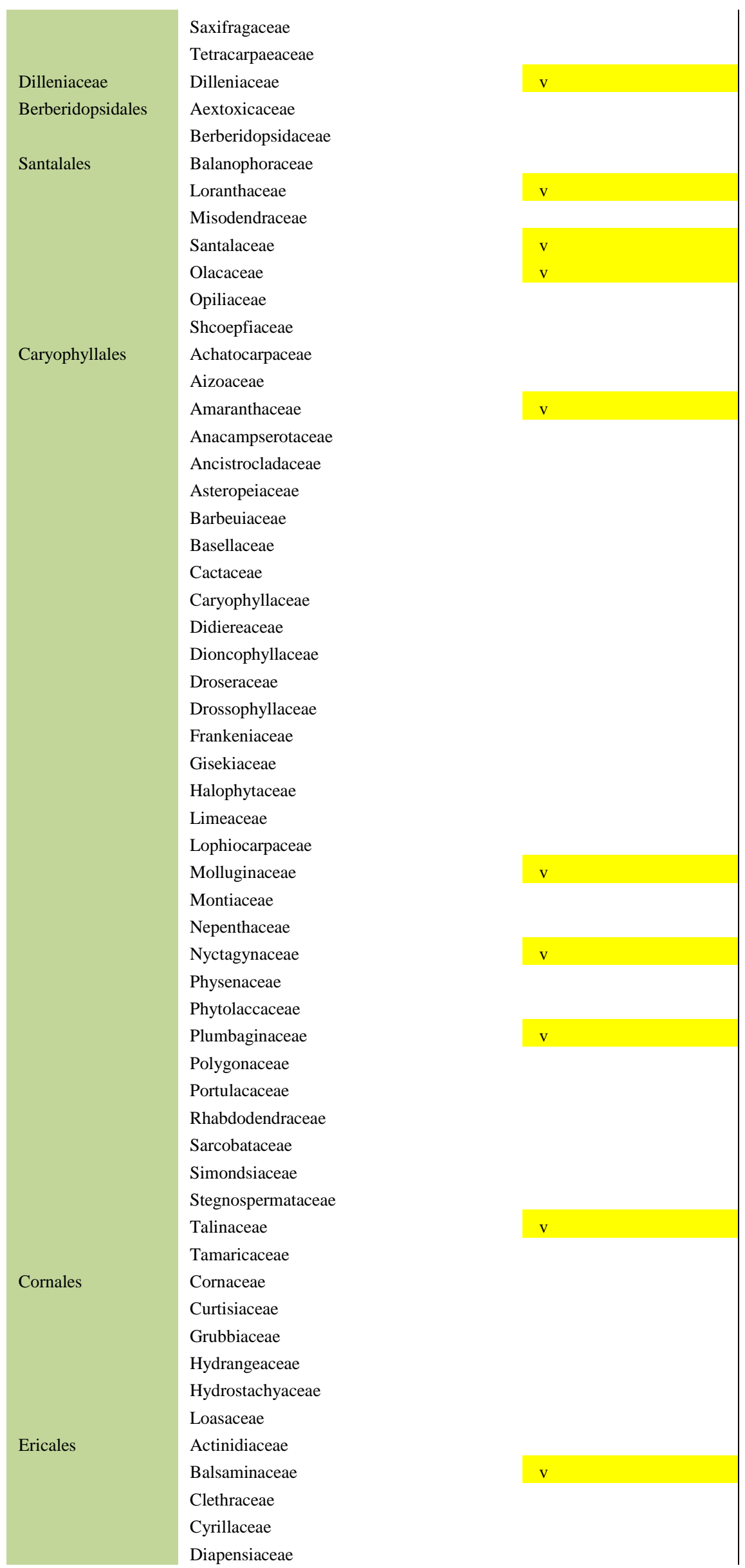
Widodo \& Luthfi - Checklist of Flowering Plants (Magnoliophyta) of Mount Nglanggeran, Gunungkidul ...

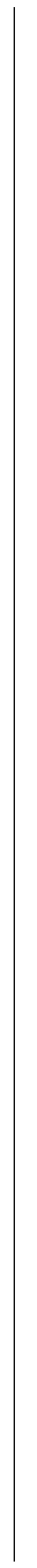

Ebenaceae

Ericaceae

Fouquieriaceae

Lecythidaceae

Maregravuaceae

Mitrastemnaceae

Pentaphylacaceae

Polemoniaceae

Primulaceae

Roridulaceae

Sapotaceae

Sarraceniaceae

Sladeniaceae

Styracaceae

Symplocaceae

Tetrameristaceae

Theaceae

\begin{tabular}{|c|c|c|c|c|}
\hline & & & & \\
\hline ASTERIDS & Lamiids & Garryales & Eucommiaceae & \\
\hline & & & Garryaceae & \\
\hline & & Gentianales & Apocynaceae & $\mathrm{v}$ \\
\hline & & & Gelsemiaceae & \\
\hline & & & Gentianaceae & $\mathrm{v}$ \\
\hline & & & Loganiaceae & $\mathrm{v}$ \\
\hline & & & Rubiaceae & $\mathrm{v}$ \\
\hline & & Lamiales & Acanthaceae & $\mathrm{v}$ \\
\hline & & & Bignoiaceae & $\mathrm{v}$ \\
\hline & & & Byblidaceae & \\
\hline & & & Calceolariaceae & \\
\hline & & & Carlemanniaceae & \\
\hline & & & Gesneriaceae & V \\
\hline & & & Lamiaceae & $\mathrm{v}$ \\
\hline & & & Linderniaceae & \\
\hline & & & Lentibulariaceae & \\
\hline & & & Martyniaceae & \\
\hline & & & Oleaceae & v \\
\hline & & & Orobanhaceae & \\
\hline & & & Paulowniaceae & \\
\hline & & & Pedaliaceae & \\
\hline & & & Phrymaceae & \\
\hline & & & Plantaginaceae & \\
\hline & & & Plocospermataceae & \\
\hline & & & Schlegeliaceae & \\
\hline & & & Scrophulariaceae & $\mathrm{v}$ \\
\hline & & & Stilbaceae & \\
\hline & & & Tetrachondraceae & \\
\hline & & & Thomandersiaceae & \\
\hline & & & Verbenaceae & $\mathrm{v}$ \\
\hline & & Solanales & Convolvulaceae & v \\
\hline & & & Hydroleaceae & \\
\hline & & & Montiniaceae & \\
\hline & & & Solanaceae & $\mathrm{v}$ \\
\hline & & & Sphenocleaceae & \\
\hline & & Boraginaceae & Boraginaceae & $\mathrm{v}$ \\
\hline & & & Vahliaceae & \\
\hline & & & Icacinaceae & \\
\hline & & & Metteniusaceae & \\
\hline & & & Oncotheaceae & \\
\hline
\end{tabular}




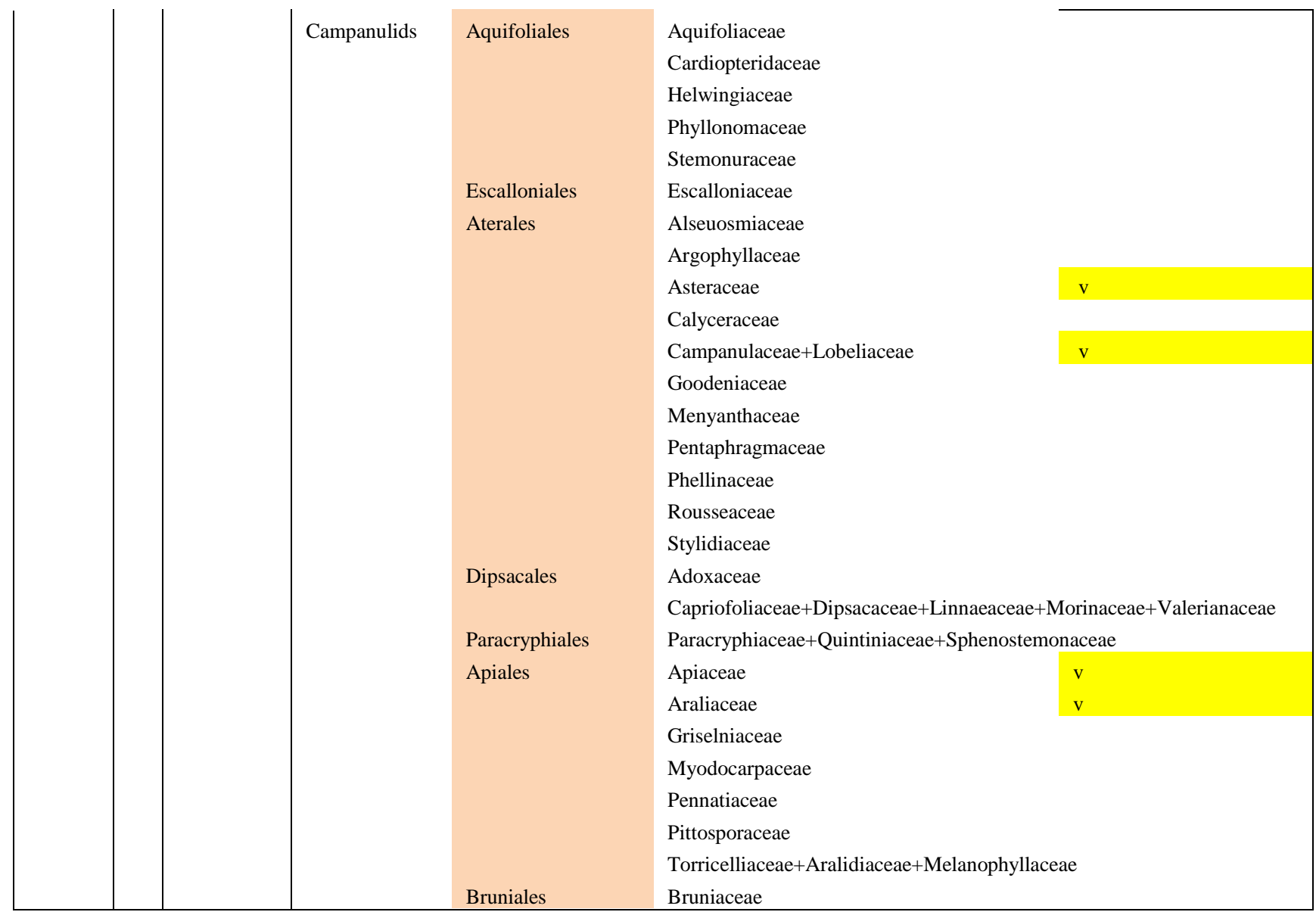

